\title{
A MULTI-MECHANISM MODEL FOR CUTTING SIMULATIONS BASED ON THE CONCEPT OF GENERALIZED STRESSES
}

\author{
C. Cheng*, R. Mahnken*, I. M. Ivanov ${ }^{\dagger}$ and E. Uhlmann ${ }^{\dagger}$ \\ * Chair of Engineering Mechanics (LTM), \\ University of Paderborn, \\ Warburgerstr. 100, 33098 Paderborn, Germany, \\ e-mail: Cheng@ltm.upb.de, web page: http://www.ltm.uni-paderborn.de/ \\ ${ }^{\dagger}$ Institute for Machine Tools and Factory Management (IWF), \\ Technical University Berlin, \\ Pascalstraße, 10587 Berlin, Germany \\ e-mail: ivanmitkov.ivanov@iwf.tu-berlin.de - Web page: http://www.iwf.tu-berlin.de/
}

Key words: generalized stresses, gradient term, additional degree of freedom, asymmetric effects, cutting simulation, phase transformation

\begin{abstract}
Based on the concept of generalized stresses as proposed by GuRTIN [6] and FOREST [4], we extend an existing model for strain rate- and temperature-dependent asymmetric plastic material behaviour accompanied by phase transformation with a gradient term of phase fraction. To this end a chemical variable, representing the austenite volume fraction, is treated as an extra degree of freedom, and the influence of its first gradient will be studied. A generalized principle of virtual power is postulated involving generalized stresses and used to derive the constitutive equations. The bulk model is formulated within a thermodynamic framework. For the scenario of a cutting process we have a martensite-austenite-martensite transformation. Finally, a cutting simulation is investigated to test our model and the different mechanisms are illustrated.
\end{abstract}

\section{INTRODUCTION}

In cutting process the work piece is machined under high speed causing highly inhomogeneous strain rates and temperature. Phase transformations are essential effects of this process due to the intense, localized, rapid thermal-mechanical loading, which affect the mechanical properties of the workpiece. For describing the material behavior, many macroscopic models were developed over the last years. DUdzInski AND MolinaRI [3] provide a simple model to consider the shearing produced during the chip formation in 
orthogonal cutting. A physically-based model for describing material behaviour is introduced by ZERILLI AND ARMSTRONG [13] based on simplified dislocation mechanics. Ductile damage at high strain-rates is considered by SIEvERT et al.[11].

GURTIN [6] formulate phase field models within a continuum thermodynamic framework, where microforces associated with the order parameter and its first gradient are introduced. Based on additional degrees of freedom and generalized stresses, ForEsT et al. [4] describe a thermodynamic consistent phase field model, which is extended with gradient terms. It is demonstrated, that there are strong links between generalized continuum mechanics and phase field models which are striving in modern field theories of materials. As a special case, the order parameter which describes phase changes in a mesoscopic modelling can be regarded as a phase fraction for macroscopic modelling.

Based on this theory, we formulate a multi-mechanism model according to [2] as an extension of our previous work [9]. To this end, the previous model is extended by an additional degree oft freedom, the phase fraction. Furthermore, we study the influence of its first gradient on the visco-plastic material behavior.

This paper is organized as follows:

- Section 2 presents a thermodynamic framework for combined visco-plasticity and phase transformations based on the concept of generalized stresses. Starting with balance relations for the generalized stresses by using the principle of virtual power, the Clausius-Duhem inequality is formulated and decomposed into an inelastic, a chemical and a thermal part.

- In the example in Section 3, cutting simulations are performed to test our extended model. To this end, the different mechanisms for cutting processes are illustrated and the influence of the extended gradient term is studied.

\section{Notations}

Square brackets [•] are used throughout the paper to denote 'function of' in order to distinguish from mathematical groupings with parenthesis $(\bullet)$.

\section{A THERMODYNAMIC FRAMEWORK FOR VISCO-PLASTICITY AND PHASE TRANSFORMATIONS BASED ON THE CONCEPT OF GEN- ERALIZED STRESSES}

\subsection{General setting}

According to Gurtin's theory [6], there exists a scalar internal microstress $\pi$ and a vector microstress $\boldsymbol{\xi}$. For a mesoscopic formulation, they perform work in conjunction with changes in the configurations of atoms, characterized by a chemical variable, the order parameter $\phi$, and its first gradient $\nabla \phi$. For the macroscopic case, the phase volume fraction $z_{A}$ is regarded as the chemical variable representing the austenite volume fraction. In this work we only concentrate on the macroscopic formulation. Considering 
the special two-phases transformation, a martensite-austenite-martensite formation of a cutting process, the martensite volume fraction is

$$
z_{M}=1-z_{A}
$$

Considering a non-isothermal case and the additional variable $z_{A}$, the degrees of freedom (DOF) are

$$
\mathrm{DOF}=\left\{\mathbf{u}, \quad \theta, \quad z_{A}\right\}
$$

A first gradient theory is built on this as

$$
\operatorname{STRAIN}=\left\{\varepsilon, \quad \theta, \quad \nabla \theta, \quad z_{A}, \quad \nabla z_{A}\right\},
$$

where $\varepsilon$, the total strain tensor, is the symmetric part of the gradient of the displacement $\mathbf{u}$, which is split as

$$
\varepsilon=\varepsilon^{e l}+\varepsilon^{\theta}+\varepsilon^{t v}+\varepsilon^{i n}
$$

Here, $\varepsilon^{e l}$ represents the elastic strain tensor, $\varepsilon^{\theta}$ the thermal expansion strain tensor, $\varepsilon^{t v}$ transformation expansion strain tensor and $\varepsilon^{i n}$ the inelastic strain tensor.

According to AMmAR et al. [1] the principle of virtual power for the macroscopic case reads

$$
\begin{aligned}
\text { 1. } \mathcal{P}^{(i)}\left[\delta z_{A}, \delta \mathbf{u}, V\right] & =\int_{V} p^{(i)}\left[\delta z_{A}, \delta \mathbf{u}\right] d v \\
\text { 2. } & \mathcal{P}^{(e)}\left[\delta z_{A}, \delta \mathbf{u}, V\right]=\int_{V} p^{(e)}\left[\delta z_{A}, \delta \mathbf{u}\right] d v \\
\text { 3. } & \mathcal{P}^{(c)}\left[\delta z_{A}, \delta \mathbf{u}, V\right]=\int_{\partial V} p^{(c)}\left[\delta z_{A}, \delta \mathbf{u}\right] d s
\end{aligned}
$$

for all subdomains $\mathcal{D}$ of the body $V$. Here $\mathcal{P}^{(i)}, \mathcal{P}^{(e)}$ and $\mathcal{P}^{(c)}$ are the overall virtual powers of internal, external and contact generalized forces, and $p^{(i)}, p^{(e)}$ and $p^{(c)}$ are related densities, respectively. $\delta z_{A}$ and $\delta \mathbf{u}$ represent the virtual volume phase fraction and the virtual displacement. With generalized stresses $\{-\pi, \boldsymbol{\xi}, \boldsymbol{\sigma}\}$ and its power-conjugates $\left\{\delta z_{A}, \nabla \delta z_{A}, \nabla \delta \mathbf{u}\right\}$ the virtual power densities of internal and external generalized forces are expressed as

$$
\begin{aligned}
\text { 1. } p^{(i)}\left[\delta z_{A}, \delta \mathbf{u}\right] & =\pi \delta z_{A}-\boldsymbol{\xi} \cdot \nabla \delta z_{A}-\boldsymbol{\sigma}: \nabla \delta \mathbf{u} \\
\text { 2. } p^{(e)}\left[\delta z_{A}, \delta \mathbf{u}\right] & =\gamma \delta z_{A}+\boldsymbol{\gamma} \cdot \nabla \delta z_{A}+\mathbf{f} \cdot \delta \mathbf{u} .
\end{aligned}
$$

Here $\boldsymbol{\sigma}$ is the Cauchy stress tensor, and $\mathbf{f}$ the volumetric density of force. The external microforces are represented by the scalar $\gamma$ and the vector $\gamma$ as introduced in GURTIN [6]. Furthermore, the virtual power density of generalized contact forces reads

$$
p^{(c)}\left[\delta z_{A}, \delta \mathbf{u}\right]=\zeta \delta z_{A}+\mathbf{t} \cdot \delta \mathbf{u},
$$


where $\zeta$ is the surface density of microtraction (a scalar) and $\mathbf{t}$ the surface density of cohesion forces (a tensor). $\zeta$ and $\mathbf{t}$ are two generalized contact forces applied to the body for the purely mechanical part over the boundary.

Assuming that no inertial microforces exist, the principle of virtual power requires, that the virtual powers of external, internal and contact forces are balanced on any subdomain $\mathcal{D} \subset V$, for any choice of the virtual phase fraction and displacement fields:

$$
\mathcal{P}^{(i)}\left[\delta z_{A}, \delta \mathbf{u}, \mathcal{D}\right]+\mathcal{P}^{(c)}\left[\delta z_{A}, \delta \mathbf{u}, \mathcal{D}\right]+\mathcal{P}^{(e)}\left[\delta z_{A}, \delta \mathbf{u}, \mathcal{D}\right]=0, \quad \forall \delta z_{A}, \forall \delta \mathbf{u}, \forall \mathcal{D} \subset V .
$$

Inserting the relations (5)-(7) into Eq.(8) yields

$$
\begin{aligned}
\int_{\mathcal{D}}(\pi+\nabla \cdot \boldsymbol{\xi}+ & \gamma-\nabla \cdot \gamma) \delta z_{A}+(\nabla \cdot \boldsymbol{\sigma}+\mathbf{f}) \cdot \delta \mathbf{u} d v \\
& +\int_{\partial \mathcal{D}}(\zeta-\boldsymbol{\xi} \cdot \mathbf{n}) \delta z_{A}+(\mathbf{t}-\boldsymbol{\sigma} \cdot \mathbf{n}) \cdot \delta \mathbf{u} d s=0 .
\end{aligned}
$$

This equation can be satisfied for any $\delta z_{A}$ and $\delta \mathbf{u}$, and $\forall \mathcal{D}$ if and only if:

$$
\begin{array}{ll}
\text { 1. } \nabla \cdot(\boldsymbol{\xi}-\boldsymbol{\gamma})+\pi+\gamma=0 \text { in } V, & \text { 2. } \zeta=(\boldsymbol{\xi}-\boldsymbol{\gamma}) \cdot \mathbf{n} \text { on } \partial V, \\
\text { 3. } \nabla \cdot \boldsymbol{\sigma}+\mathbf{f}=0 \text { in } V, & \text { 4. } \mathbf{t}=\boldsymbol{\sigma} \cdot \mathbf{n} \text { on } \partial V .
\end{array}
$$

Eq.(10.1) and Eq.(10.3) represent the balance equations associated with phase volume fraction $z_{A}$ and displacement $\mathbf{u}$, respectively. Eq.(10.2) and Eq.(10.4) express boundary conditions for the generalized microtration $\zeta$ and the cohesion forces $\mathbf{t}$, respectively.

For the sake of brevity we assume $\gamma=0$ for the scalar external microforce and $\gamma=\mathbf{0}$ for the vector external force. Therefore, from Eq.(10.1) we obtain the relation

$$
\nabla \cdot \boldsymbol{\xi}+\pi=0 \text { in } V \text {. }
$$

The local balance of energy and the entropy principle are given by, see FOREST et al. $[5]$

$$
\begin{aligned}
& \text { 1. } \quad \rho \dot{\epsilon}+\operatorname{div} \mathbf{q}=p^{(i)}+\rho r_{\theta} \quad \text { in } V \text {, } \\
& \text { 2. }-\rho \dot{\Psi}+\rho \theta \dot{\eta}+p^{(i)}-\frac{1}{\theta} \mathbf{q} \cdot \nabla \theta \geq 0 . \quad \text { in } V \text {. }
\end{aligned}
$$

In addition to the above notations we use: $\rho$ - density, $\epsilon$ - specific internal energy, $\mathbf{q}$ heat-flux density vector, $r_{\theta}$ - mass density of heat supply. Analogously to Eq.(6.1) the density of internal power is introduced by FOREST et al. [5] as

$$
p^{(i)}\left[\dot{z}_{A}, \dot{\mathbf{u}}\right]=-\pi \dot{z}_{A}+\boldsymbol{\xi} \cdot \nabla \dot{z}_{A}+\boldsymbol{\sigma}: \dot{\boldsymbol{\varepsilon}}
$$

which is an extension of a standard formulation, see e.g. HAUPT [7]. 


\subsection{Constitutive Framework}

The Helmholtz free energy $\Psi$ is assumed as

$$
\Psi=\Psi\left[\varepsilon^{e l}, \underline{a}, z_{A}, \nabla z_{A}, \theta\right],
$$

where $\varepsilon^{e l}$ is the elastic strain tensor, $\underline{a}=\left[a_{i}, \ldots, a_{n_{a}}\right]$ is a vector of internal variables for strain hardening. Next, we introduce constitutive relations for the Cauchy stress tensor $\boldsymbol{\sigma}$, the vector microstress $\boldsymbol{\xi}$ and the entropy $\eta$, and define thermodynamic forces $\underline{A}=\left[A_{1}, A_{2}, \ldots, A_{n_{a}}\right]^{T}$ and chemical force $Z_{A}$

$$
\begin{aligned}
& \text { 1. } \boldsymbol{\sigma}=\rho \frac{\partial \Psi}{\partial \varepsilon^{e l}}, \text { 2. } \quad \boldsymbol{\xi}=\rho \frac{\partial \Psi}{\partial \nabla z_{A}}, \quad 3 . \quad \eta=-\frac{\partial \Psi}{\partial \theta}, \\
& \text { 4. } \underline{A}=\rho \frac{\partial \Psi}{\partial \underline{a}}, \quad \text { 5. } \quad Z_{A}=\rho \frac{\partial \Psi}{\partial z_{A}}+\pi .
\end{aligned}
$$

The thermodynamic forces $A_{i}$ are also called hardening stresses. The relations (15.1) and (15.3) result from the Clausius-Duhem inequality by standard arguments, see e.g. [10]. The following inequalities are sufficient for validity of the Clausius-Duhem inequality $(12.2)$ :

$$
\text { 1. } \mathcal{D}^{i}=\boldsymbol{\sigma}: \dot{\varepsilon}^{i n}-\underline{A} \underline{\dot{a}} \geq 0, \quad \text { 2. } \mathcal{D}^{z}=-Z_{A} \dot{z}_{A} \geq 0, \quad \text { 3. } \mathcal{D}^{\theta}=-\frac{1}{\theta} \mathbf{q} \cdot \nabla \theta \geq 0 .
$$

\section{SIMULATION OF A CUTTING PROCESS}

In this example a cutting process is investigated in order to test our model. For consideration of the extra degree of freedom $z_{A}$, the model must be implemented on the element level. To this end, the model is implemented as a user-defined element subroutine (VUEL) and linked to ABAQUS v6.11. Furthermore, in order to capture the separation effects (element deletion) for the cutting process, an explicit algorithm on the (global) FiniteElement level is used. Moreover, large strain modelling is taken into account using an objective integration algorithm. This method is used to obtain correct rotational transformation properties as presented by Hughes AND Winget [8]. For more details on the objective integration algorithm we refer to [2].

Figure 1 shows the geometry and the finite-element discretization. The tool has a negative rake angle $\alpha=-6^{\circ}$ and a clearance angle $\beta=6^{\circ}$. The workpiece (2D) is 2000 $\mu \mathrm{m}$ long and $400 \mu \mathrm{m}$ high. The boundary conditions on the workpiece are shown in Figure 1. A separation layer with only one thin element in vertical direction is defined for chip formation during the cutting simulation. To this layer we assign a simple failure criterion $e_{v} \leq \mathrm{e}_{v}^{f}$ ( $e_{v}^{f}$ is a constant) in dependence of the effective plastic strain $e_{v}$ for separating the chip from the workpiece. In this way the separation layer can be deleted during the simulation, which means, that the surface of separation is prescribed. For 
simplicity, the elements below the separation layer can not move vertically, and we do not consider friction effects on the cutting surface. The tool moves in horizontal direction with constant velocity $\mathrm{v}=3.5 \mathrm{~m} / \mathrm{s}$. Room temperature is applied at the beginning of the simulation, and for cooling a conventional temperature condition is applied over the surfaces of the workpiece.

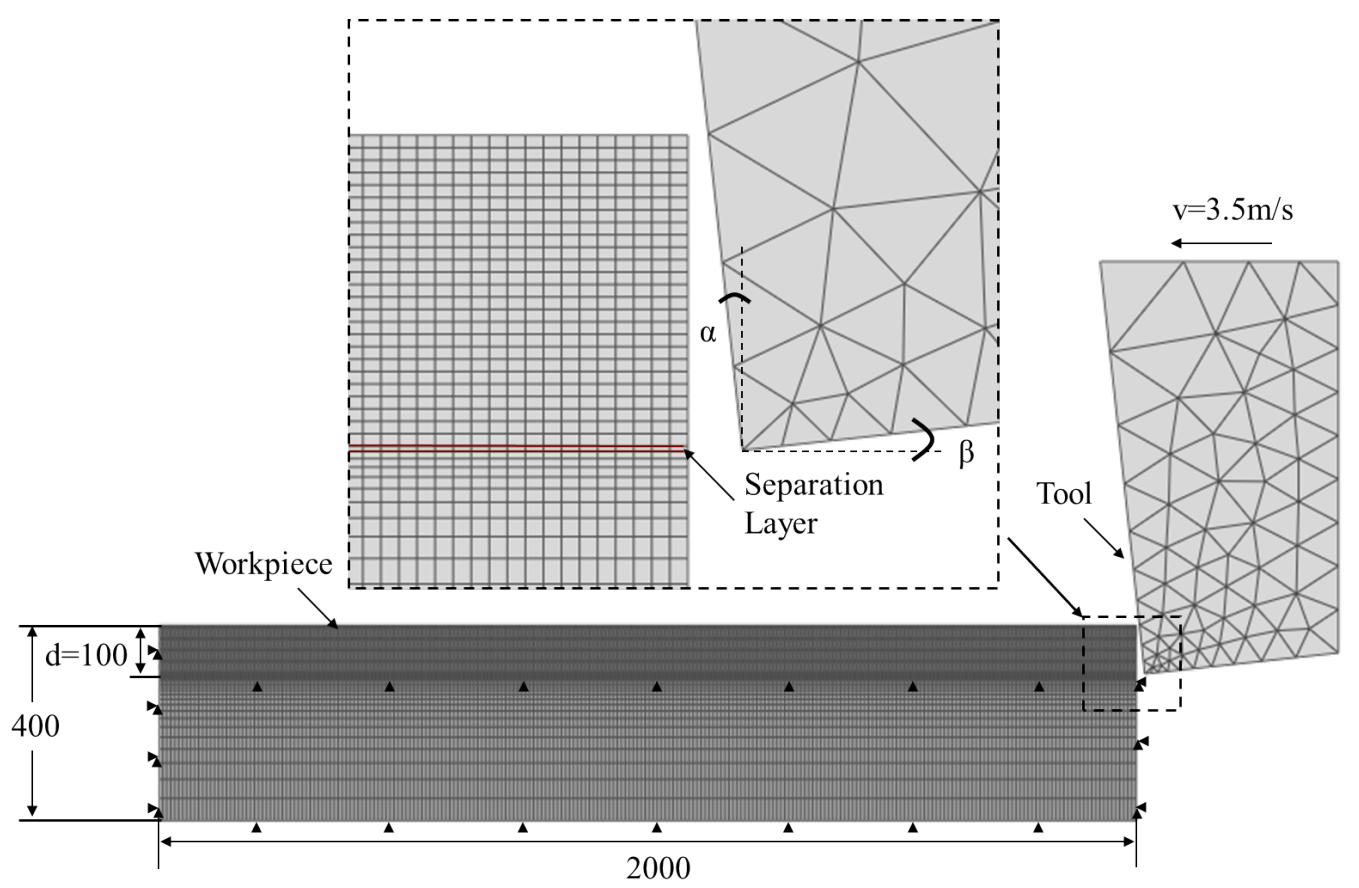

Figure 1: Cutting simulation: Geometry and finite-element discretization

The results are presented in Figure 2. a) and b) show that high stresses and temperatures appear at the cutting edge, and high residual stresses at the chip top. c) shows different stress zones indicated by the stress mode factor, which is introduced in [9], for consideration of the asymmetric effects. Furthermore, the influence of the gradient term for phase transformation is demonstrated. d), e) and f) show the austenite phase fraction (the rectangle in $\mathrm{d}$ ) visualises a representative volume element for the next example on mesoscopic modelling), its gradient in horizontal and vertical direction without influence of the gradient term. In comparison, g), h) and i) are under the influence of the gradient term. The austenite phase fraction and its gradients are clearly different because of the influence of the extended gradient term. In the summary in Section 4 we comment on future challenges regarding the gradient term and its identification. 
a)

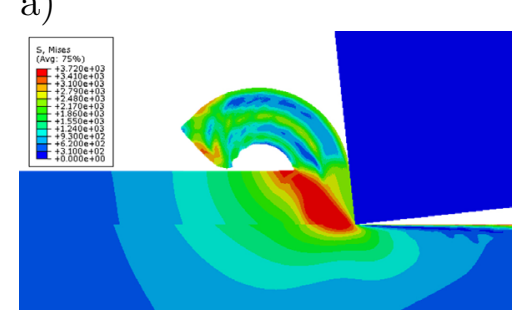

d)

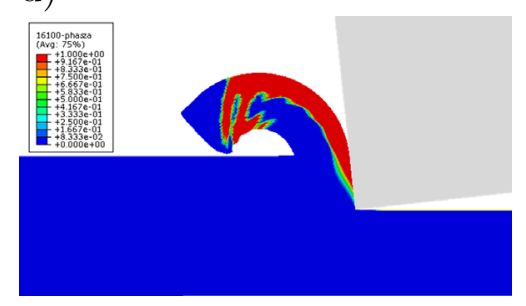

g)

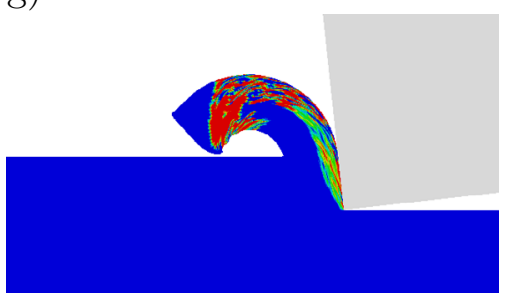

b)

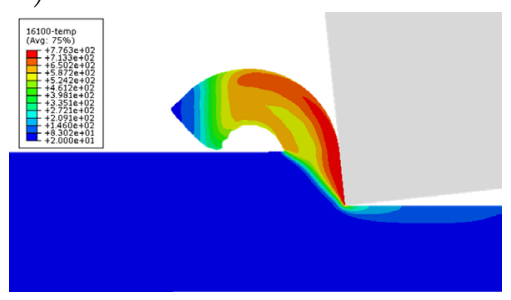

e)

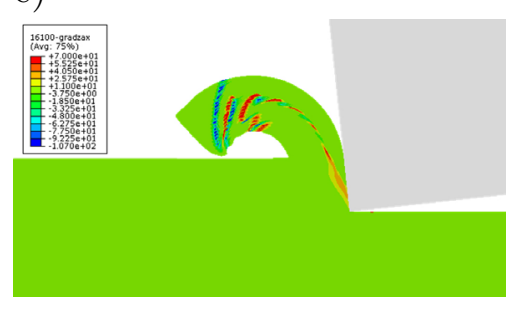

h)

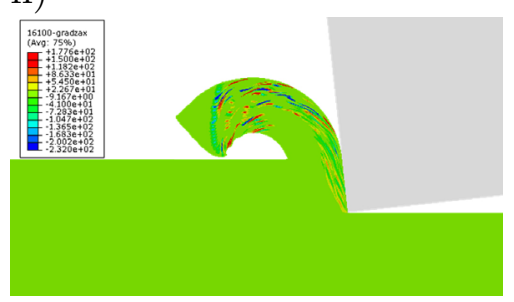

c)

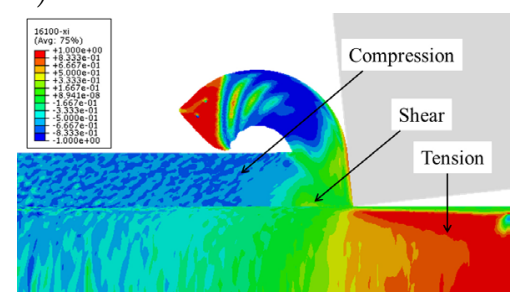

f)

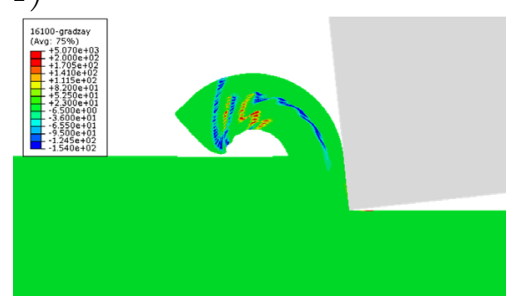

i)

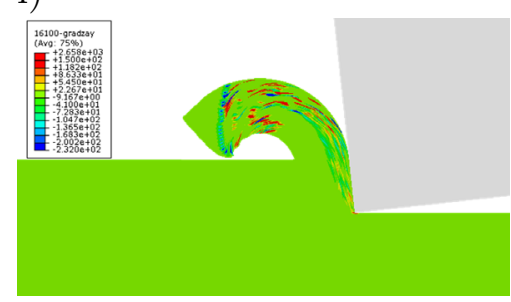

Figure 2: Cutting simulation: Contours of a) von-Mises stress, b) temperature, c) stress mode factor, d) and g) austenite volume fraction(the rectangle in d) visualise a representative volume element for the next example on mesoscopic modelling), e) and h) gradient of $z_{A}$ in horizontal direction, f) and i) gradient of $z_{A}$ in vertical direction, d), e) and f) show without, and g), h) and i) with influence of the gradient term

\section{SUMMARY AND CONCLUSIONS}

Based on the concept of generalized stresses in ForEST et al. [4], we extended a multimechanism model developed in our previous work [9] with a gradient term. This general model has been specialized to the scenario of a cutting process in steel production. Furthermore, the model has been implemented as a user-defined subroutine linked to Abaqus and was applied for a cutting simulation. At last, the influence of the gradient term was studied.

So far, the model is formulated within a small strain theory, and large strain modelling required for the cutting simulation is considered using an objective integration algorithm. A formulation of the model in a large deformation theory is an essential work for the future. Furthermore, an interesting challenge is the identification of the gradient term and the related parameter $\alpha$ on the basis of experiments. The studied material DIN $100 \mathrm{Cr} 6$ has a carbon content from 0.93 to $1.05 \%$, which leads to a stabilisation of austenit 
after the cooling in a martensite-austenite-martensite transformation during a cutting process. The quantities and space distribution of the rest austenite can be measured by using Electron Backscatter Diffraction (EBSD). According to this aspect we intend to identify the parameter $\alpha$. Moreover, the multi-mechanism model will be extended with hardness dependency according to UMBRELLO et al. [12].

\section{ACKNOWLEDGEMENT}

This paper is based on investigations of the SPP 1480 'Modellierung, Simulation und Kompensation von thermischen Bearbeitungseinflüssen für komplexe Zerspanprozesse', which is kindly supported by the Deutsche Forschungsgemeinschaft (DFG).

\section{REFERENCES}

[1] Ammar, K.; Appolaire, B.; Cailletaud, G.; Feyel, F.; and Forest, S., 2009, Finite element formulation of a phase field model based on the concept of generalized stresses, Computational Materials Sciences, (45)800-805

[2] Cheng, C., Mahnken, R., 2015, A multi-mechanism model for cutting simulations based on the concept of generalized stresses, Comp. Mat. Sci., 100, B, 144-158

[3] Dudzinski, D., Molinari, A., 1997, Modelling of cutting for viscoplastic materials, Int. J. Mech. Sci., 39, 4, 369-389

[4] Forest, S.; Ammar, K.; Appolaine, B., 2011, Micromorphic vs. Phase-Field Approaches for gradient Viscoplasticity and Phase Transformations, Lecture Notes in Applied and Computational Mechanics 59 (2011), S. 69 - 88

[5] Forest, S.; Aifantis, E. C., 2011, Some links between recent gradient thermoelasto-plasticity theories and the thermomechanics of generalized continua, International Journal of Solids and Structures 47 (2010), S. 3367 - 3376

[6] Gurtin, M., 1996, Generalized Ginzburg-Landau and Cahn-Hilliard equations based on a microforce balance, Physica D, 92:178-192

[7] Haupt, P., 2002, Continuum Mechanics and Theory of Materials, Springer-Verlag, Berlin

[8] Hughes, T. J. R.; Winget, J., 1980, Finite rotation effects in numerical integration of rate constitutive equations arising in large-deformation analysis, Int. J. numer. Meth. Engng, 15, 1862-1867

[9] Mahnken, R.; Wolff, M.; Cheng, C., 2013, A multi-mechanism model for cutting simulations combining visco-plastic asymmetry and phase transformation, Int. J. Solids Struct. Vol. 50, S. 3045-3066 
[10] Mahnken, R.; Wolff, M.; Schneidt, A.; Böhm, M., Multi-Phase Transformations at Large Strains - Thermodynamic Framework and Simulation, International Journal of Plasticity 39 (2012) 1-26

[11] R. Sievert, H.-D. Noack, A. Hamann, P. Lwe, K.N. Singh, G. Knecke, R. Clos, U. Schreppel, P. Veit, E. Uhlmann, R. Zettier, 2003, Simulation der Spansegmentierung beim Hochgeschwindigkeits- Zerspanen unter Berücksichtigung duktiler Schädigung, Technische Mechanik, 23, 2-4, 216-233

[12] Umbrello, D.; Hua, J.; Shivpuri, R., 2004, Hardness-based flow stress and fracture models for numerical simulation of hard machining AISI 52100 bearing steel, Ma. Sci. Eng, A 374, 90-100

[13] Zerilli, F.J.; Armstrong, R.W., 1987, Dislocation-mechanics-based constitutive relations for material dynamics calculations, Journal of Applied Physics, 61, (5): 1816 